\title{
Topographical differences of frontal-midline theta activity reflect functional differences in cognitive control abilities
}

\author{
Kathrin C. J. Eschmann ${ }^{1}$, Regine Bader ${ }^{1}$, \& Axel Mecklinger ${ }^{1}$ \\ ${ }^{1}$ Experimental Neuropsychology Unit, Department of Psychology, Saarland University, \\ Saarbrücken, Germany
}

Corresponding Author:

Kathrin C. J. Eschmann

Experimental Neuropsychology Unit

Department of Psychology, Saarland University

Campus Building A2 4, Room 2.15

66123 Saarbrücken, Germany

Phone: +49 681-30262868

e-Mail: kathrin.eschmann@uni-saarland.de 


\section{Abstract}

Electrophysiological oscillations are assumed to be the core mechanism for large-scale network communication. The specific role of frontal-midline theta oscillations as cognitive control mechanism is under debate. According to the dual mechanisms of control framework, cognitive control processes can be divided into proactive and reactive control. The present study aimed at investigating the role of frontal-midline theta activity by assessing oscillations in two tasks varying in the type of cognitive control needed. More specifically, a delayed match to sample (DMTS) task requiring proactive control and a color Stroop task recruiting reactive control processes were conducted within the same group of participants. Moreover, both tasks contained conditions with low and high need for cognitive control. As expected larger frontal-midline theta activity was found in conditions with high need for cognitive control. However, theta activity was focally activated at frontal sites in the DMTS task whereas it had a broader topographical distribution in the Stroop task, indicating that both proactive and reactive control are reflected in frontal-midline theta activity but reactive control is additionally characterized by a broader theta activation. These findings support the conclusion that frontal-midline theta acts functionally different depending on the task requirements.

Keywords: frontal-midline theta, cognitive control, working memory, interference 


\section{Introduction}

Cognitive control processes are needed to function in everyday life and are assumed to be reflected in theta activity (ca. 4-7 Hz) that mainly occurs at frontal midline electrode sites (see Cavanagh \& Frank, 2014; Sauseng, Griesmayr, Freunberger, \& Klimesch, 2010, for review). Although frontal midline (FM) theta has been found in numerous studies investigating cognitive control abilities, its precise functional role is still under debate. In a recent comprehensive review, Cavanagh and Frank (2014) proposed that FM theta represents a general control mechanism that reflects the need for and the implementation of cognitive control. This assumption is based on observations of goal-directed or habituating behavior, in which cognitive control processes are needed in order to resolve a situation with the best possible outcome and to adaptively optimize performance for future encounters of similar situations. The assumption of a general control mechanism (Cavanagh \& Frank, 2014) receives support by several electrophysiological studies investigating cognitive effort in a large variety of context situations, such as during WM encoding and maintenance or episodic memory encoding and retrieval. For instance, both stimulus- and response-locked event-related potential (ERP) components that are elicited by novelty, conflict, errors or negative feedback are accompanied by increased FM theta activity (Cavanagh, Zambrano-Vazquez, \& Allen, 2012). Moreover, in working memory (WM) studies, FM theta power was shown to increase in conditions with high WM load and task difficulty (Gevins, Smith, McEvoy, \& Yu, 1997; Griesmayr, Gruber, Klimesch, \& Sauseng, 2010; Jensen \& Tesche, 2002; Onton, Delorme, \& Makeig, 2005; Roberts, Hsieh, \& Ranganath, 2014; Wilson, Swain, \& Ullsperger, 1999; see Sauseng et al., 2010, for review). In some of these studies, the increase in theta activity during WM was also predictive of later long-term memory retrieval (Gruber, Tsivilis, Giabbiconi, \& Müller, 2008; Khader, Jost, Ranganath, \& Rösler, 2010; Osipova et al., 2006; Sederberg, Kahana, Howard, Donner, \& Madsen, 2003; see Nyhus \& Curran, 2010, for review) suggesting that FM theta reflects cognitive control processes that serve both WM and episodic memory functions. In contrast to WM memory studies, difficulty of episodic memory retrieval can lead to both, increases and decreases of FM theta, suggesting that FM theta reflects different aspects 
of episodic memory, such as memory representation strength or memory evaluation processes (Klimesch et al., 2006). FM theta power was also shown to reflect the amount of cognitive control recruitment in interference situations in which two contrary responses are in conflict to each other, such as in the incongruent trials of a Stroop, Simon and flanker task or No-Go trials in a Go/No-Go task (HansImayr et al., 2008; Nigbur, Ivanova, \& Stürmer, 2011). Additionally, FM theta activity declines with increasing interference resolution in competitive memory retrieval (Ferreira, Marful, Staudigl, Bajo, \& Hanslmayr, 2014; Spitzer, Hanslmayr, Opitz, Mecklinger, \& Bäuml, 2009; Waldhauser, Bäuml, \& Hanslmayr, 2014). In these situations, memory representations, which either belong to the same category or are associated with each other, compete for retrieval. While the interference induces FM theta activity, the successful suppression of competing associations leads to a decrease in FM theta activity.

FM theta oscillations are assumed to communicate and implement the need for cognitive control in different neural systems comprising both neighboring as well as distant brain regions (Cavanagh \& Frank, 2014; Cavanagh et al., 2012; Helfrich \& Knight, 2016). Thereby, the synchronization of neurons that belong to assumed FM theta source regions, such as the anterior cingulate cortex (ACC) or the midcingulate cortex (MCC), leads to FM theta amplitudes that provide temporal windows for segregating information intake via corresponding activity of other cortical populations (Cavanagh \& Frank, 2014). The coincident activation between FM theta source regions and other task-relevant brain regions is reflected in theta phase synchronization and can be interpreted as information intake and transfer between these regions (Asada, Fukuda, Tsunoda, Yamaguchi, \& Tonoike, 1999; Cohen, 2011; Gevins et al., 1997; Onton et al., 2005). For instance, situations, in which an unexpected feedback or conflict occurs, are characterized by theta phase synchronization between areas that play an important role in conflict detection and the lateral prefrontal cortex (IPFC), an area critical for active task-goal maintenance (Cavanagh, Frank, Klein, \& Allen, 2010). Accordingly, information about the detected conflict is communicated and leads to the adaptive modification of task-goals. In contrast, in order to prevent response errors in the future, 
mid-frontal brain areas synchronize with occipital sensory regions after an erroneous response in a No-Go trial (Cohen, van Gaal, Ridderinkhof, \& Lamme, 2009). This theta phase synchronization between mid-frontal and occipital areas after an error even persists into following trials. It is assumed that mid-frontal brain regions thereby exert top-down control over stimulus processing that happens in sensory areas. Consistent with this view, the theta phase synchronization between midfrontal brain regions and motor areas found in humans and in rats is interpreted to reflect the control over conflicting motor responses (Narayanan, Cavanagh, Frank, \& Laubach, 2013). All in all, although different brain regions synchronize in different tasks serving different control mechanisms due to different task demands, the neural activity reliably results in FM theta activity measured at mid-frontal sites (see Cavanagh \& Frank, 2014, for review).

The aforementioned studies mainly focused on cognitive control processes that are recruited after events with enhanced need for control in order to adaptively modify behavior. The dual mechanisms of control (DMC) framework by Braver (2012) distinguishes these forms of reactive control from proactive control. Proactive control reflects an early selection process that supports the facilitated processing of task-relevant information in a top-down manner. This is achieved by the active maintenance of task-goal information in order to bias sensory processing before the occurrence of a cognitively demanding event. The maintenance of task-goals and task-contexts is assumed to be reflected in sustained IPFC activation. In line with this assumption several brain imaging studies could show sustained IPFC activation during WM maintenance (Braver \& Bongiolatti, 2002; Braver \& Cohen, 2001; Paxton, Barch, \& Racine, 2007; Veltman, Rombouts, \& Dolan, 2003). In contrast, reactive control is defined as a late correction process that is initiated when interference is detected and a prepotent response has to be inhibited in favor of a less prepotent one. According to the DMC framework, this reactive control is anchored in the IPFC and the ACC but also recruits a wider brain network compared to proactive control (Braver, 2012). Brain imaging studies investigating the reactive control mechanisms in color Stroop tasks could show simultaneous activation of prefrontal brain areas, such as the IPFC, and parietal regions (Grandjean et al., 2012; 
Milham et al., 2002; Zysset, Mu, Lohmann, \& Cramon, 2001; see Niendam et al., 2012, for review). It is assumed that in the case of reactive control the processing of sensory or episodic memory information first leads to activation of posterior brain regions followed by conflict detection by the ACC before top-down control is exerted by PFC regions. In addition to imaging studies, studies investigating theta phase coherence between frontal and parietal sites could show the activation of differential fronto-parietal theta networks in proactive and reactive control tasks (Cooper et al., 2015; Cooper, Wong, McKewen, Michie, \& Karayanidis, 2017). However, for the analysis of proactive control, these studies focused on transient changes in proactive control for trial-type preparation that might involve a different control network than sustained maintenance of task-goals or stimuli (Cooper et al., 2015). In sum, previous research showed that proactive and reactive control processes are reflected in the activation of different networks, suggesting a smaller proactive control network including the IPFC and a wider reactive control network including frontal and parietal regions. Consequently, the question arises whether proactive and reactive control processes are also accompanied by scalp topographical differences of theta oscillations that become visible by directly comparing two tasks differing in the recruited cognitive control processes.

The present study aims at investigating whether different forms of FM theta activity are recruited in two cognitive control tasks that are characterized by either proactive or reactive control demands. For this purpose, a delayed match to sample (DMTS) task and a color Stroop task, that were part of a larger study including also an episodic retrieval task, were analyzed. Although the study was not initially designed to compare proactive and reactive control processes, the DMTS and Stroop task were assumed to differentially recruit either proactive or reactive control. In the DMTS task, participants have to maintain or manipulate a stimulus over a delay period in order to compare it to an expected probe afterwards. This task is assumed to mainly involve the proactive control mechanism that supports the sustained and anticipatory maintenance of goal-relevant information. In contrast, in the color Stroop task, participants have to inhibit the prepotent response of reading the written color word that interferes with the task goal of naming the color of the ink. This is 
expected to primarily recruit the reactive control mechanism that supports the suppression of the strongly activated task-irrelevant reading response and the strengthening of the weakly activated but task relevant color-naming response. In order to show that the degree of cognitive control recruitment has a behavioral and electrophysiological effect irrespective of the elicited cognitive control mode, both tasks contained a number of conditions differing in task difficulty and thus in the amount of cognitive control needed for performance of the respective condition. Based on previous findings, we expected slower reaction times (RTs) and lower accuracy in the conditions with high cognitive control compared to those with low cognitive control demands in both tasks. Additionally, stronger recruitment of cognitive control should be reflected in larger FM theta activation in the more difficult conditions of both tasks. Due to the fact that the cognitive control tasks differed in their proactive and reactive control demands, it was expected that the recruitment of differential cognitive control mechanisms would lead to scalp topographical differences of theta activity between both tasks.

\section{Methods}

\subsection{Participants}

Overall, 34 right-handed German volunteers who were recruited from Saarland University's student community participated in the study. For analyses of the Stroop task, data of all 34 participants (11 male, $M_{\mathrm{age}}=23.15$ years, age range $=19-27$ years) was used. For analyses of the DMTS task, seven participants had to be excluded due to chance performance (determined by individual $\chi^{2}$ tests for each participant), resulting in a sample of 27 participants ( 9 male, $M_{\text {age }}=22.81$ years, age range $=19-27$ years). Consequently, as the comparison of theta activity between tasks was within-participants, we excluded the same seven participants also from the task comparison analyses. According to self-report all participants were healthy, had normal or corrected-to-normal vision and no history of neurological or psychiatric problems. Participants provided written informed consent prior to the experiment and either received course credit or were paid $8 €$ per hour in return for their 
participation. The experimental procedure was approved by the local ethics committee in accordance with the declaration of Helsinki.

\subsection{Experimental Procedure}

Participants were seated comfortably in a dimly lit and quiet experimental room in order to conduct a DMTS task adapted from Griesmayr et al. (2014) and a color Stroop task on a Dell Computer. Experimental stimuli were presented using E-Prime 2.0 software on a Dell 24-inch monitor placed at a viewing distance of approximately $70 \mathrm{~cm}$. The order of DMTS and Stroop task was counterbalanced across participants. Both tasks were part of a larger study. Before performing the two tasks reported here, participants performed the learning phase of a source memory task, in which they learned 200 concrete nouns by judging the object denoted by the noun by different characteristics.

The DMTS task consisted of two conditions including 12 practice and 70 task trials each (Figure 1). Both conditions were presented in a blocked and counterbalanced design. Each task block consisted of 35 trials. Stimuli consisted of one or four colored squares integrated in a $6 \times 6$ matrix with a visual angle of $9.8^{\circ} \times 9.8^{\circ}$ and were presented for $500 \mathrm{~ms}$. These encoded stimuli had to be maintained over a $2000 \mathrm{~ms}$ delay period in which the stimuli were masked by a matrix containing gradational grey colored squares. In the retention condition participants had to simply maintain the location of a green-colored square within the $6 \times 6$ matrix over the delay period whereas in the manipulation condition the participants had to mentally mirror four red squares on the vertical matrix midline and maintain their mirrored locations. After the delay period, a probe matrix with grey squares either matching or not matching the position of the colored squares of the encoded stimulus was presented for 2000 ms. Participants were instructed to indicate by a mouse button press with the left or right thumb whether the grey squares of the probe were identical (match) or different (non-match) to the colored squares locations from the encoded stimulus. In the manipulation condition only one of the four squares did not match the correct mirrored position in the non-match trials. For half of the trials the correct response was match and for the other half non- 
match. Participants were asked to respond as fast and accurately as possible during the presentation of the probe. During a subsequent jittered inter-trial interval of $1500-2000 \mathrm{~ms}$ a central fixation cross was presented. All instructions and stimuli were presented centrally against a grey background.

\section{DMTS task}

retention condition
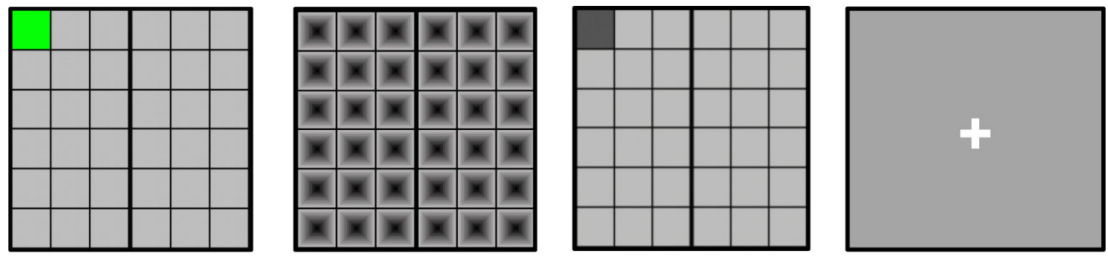

manipulation condition
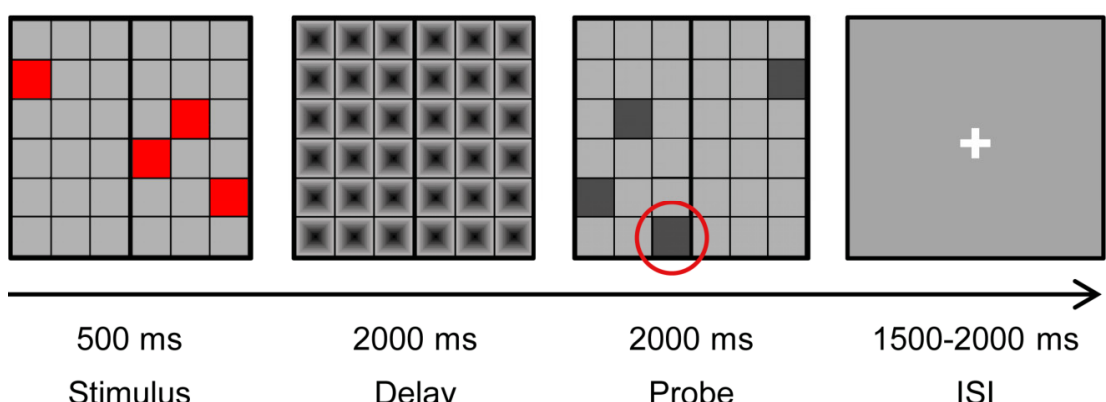

$$
\begin{gathered}
2000 \mathrm{~ms} \\
\text { Delay }
\end{gathered}
$$

$$
\begin{gathered}
2000 \mathrm{~ms} \\
\text { Probe }
\end{gathered}
$$

1500-2000 ms

ISI

Figure 1. Trial procedure of the DMTS task. In the retention condition the stimulus had to be simply maintained whereas in the manipulation condition the colored squares of the stimulus had to be mirrored at the vertical midline. The red circle, which was not shown during the experiment, indicates the square of the probe that is in the wrong position, making the depicted trial a non-match.

The color Stroop task contained a congruent, incongruent and neutral condition. Stimuli were presented in randomized order within eight blocks consisting of 24 stimuli each. Before the processing of the 192 task trials, participants could familiarize with the task in 24 practice trials. All words were presented with a visual angle of $4.1^{\circ} \times 1.2^{\circ}$ for $1000 \mathrm{~ms}$ against a black background and were preceded by a fixation cross which was presented for $1000 \mathrm{~ms}$. In the congruent condition the German color words for green ("GRÜN"), red ("ROT"), blue ("BLAU"), and yellow ("GELB") were presented in their respective ink color whereas in the incongruent condition the color words were shown in a color different from their meaning. For the neutral condition the four neutral German 
words for leak (“LECK"), oath (“EID"), joke ("WITZ"), and misfortune ("PECH") were used. These words were selected due to comparable length and because they did not share initial letters with the color words. Participants were instructed to indicate the ink color as fast and correctly as possible while ignoring the word meanings. Responses were given by pressing the colored keys " $\mathrm{C}$ " and " $\mathrm{S}$ " with the left and " $\mathrm{M}$ " and " $\mathrm{L}$ " with the right index and middle finger on a conventional keyboard. The color to key assignment was fixed across participants and experimental blocks.

\subsection{Data Acquisition and Processing}

Electroencephalographic (EEG) activity was recorded continuously with a sampling rate of $500 \mathrm{~Hz}$ from $32 \mathrm{Ag} / \mathrm{AgCl}$ electrodes using Brain Vision Recorder software and Brain Amp EEG amplifiers (Brain Products GmbH, Gilching, Germany). Scalp electrodes were mounted on a fitted cap according to the extended 10-20 system and four additional electrodes placed at the outer canthi of the eyes and above and below the right eye were used to record the electrooculogram for eye movement detection. During the continuous EEG recording, electrode impedances were kept below $5 \mathrm{k} \Omega$ and signals were filtered with an online low-pass filter of $100 \mathrm{~Hz}$. All electrodes were referenced to the left mastoid electrode during data acquisition and re-referenced offline to averaged mastoids.

Offline analysis of EEG signals was performed using Brain Vision Analyzer 2.1 software (Brain Products GmbH, Filching, Germany). Raw data was first inspected visually and nonstereotypic activity, such as muscle activity, was manually removed in order to improve artifact correction by ICA decomposition. Afterwards data was high-pass filtered at $0.5 \mathrm{~Hz}(12 \mathrm{~dB} / \mathrm{oct})$ and low-pass filtered at $40 \mathrm{~Hz}(24 \mathrm{~dB} /$ oct). An infomax ICA was run to identify and remove components representing eye movements, such as blinks and saccades. The continuous EEG of the DMTS task was segmented from $-1500 \mathrm{~ms}$ prior to stimulus presentation until $4000 \mathrm{~ms}$ thereafter and in the color Stroop task from $1500 \mathrm{~ms}$ prior to word presentation until $2500 \mathrm{~ms}$ thereafter. Finally, segments with a maximal difference of $200 \mu \mathrm{V}$ were removed, resulting in comparable trial numbers between both tasks $\left(M_{D M T S}\right.$ $\left.=58.89, S D_{D M T S}=3.75 ; M_{\text {Stroop }}=60.84, S D_{\text {Stroop }}=5.75\right)$. 
For calculation of frequency amplitude estimates, single trial activity from $1 \mathrm{~Hz}$ to $30 \mathrm{~Hz}$ was decomposed using a complex Morlet wavelet with 59 linear $0.5 \mathrm{~Hz}$ frequency steps and a timefrequency resolution indicated by a parameter c of 10. Subsequently, event-related synchronization / desynchronization (ERS/ERD) was calculated for every condition separately. ERS/ERD is defined as the percent power change relative to a baseline, which was the time interval of $-800 \mathrm{~ms}$ to $-200 \mathrm{~ms}$ before stimulus onset for both tasks. In order to exclude distortions of lower frequency power changes by ERPs, ERS/ERD was calculated with the inter-trial variance approach that calculates the frequency power by referencing the power of each measurement point within a trial to the mean power of the according measurement point of all trials (Kalcher \& Pfurtscheller, 1995). In this way, purely non-phase locked (induced) activity that is thought to reflect higher order processes is measured (David, Kilner, \& Friston, 2006). ERS/ERD measures of theta activity in both tasks were taken from electrode Fz because condition effects of the DMTS and Stroop task were shown to be present at this electrode before (cf. Griesmayr et al., 2014, 2010; Hanslmayr et al., 2008). Moreover, theta activity was largest at $\mathrm{Fz}$ for the more difficult condition of each task (manipulation condition of the DMTS task and incongruent condition of the Stroop task). Consistent with previous studies, five consecutive epochs with a length of $500 \mathrm{~ms}$ in the theta frequency range $(4-7 \mathrm{~Hz})$ at electrode $\mathrm{Fz}$ were extracted covering both the 500 ms encoding and 2000 ms maintenance phase of the DMTS task (Berger et al., 2016; Griesmayr et al., 2014). In contrast, theta effects in Stroop tasks in previous studies were shown to have a shorter temporal extension and occur during the later stimulus presentation phase (Hanslmayr et al., 2008), where also corresponding ERP effects are present (Rebai, Bernard, \& Lannou, 1997). Thus, two consecutive $400 \mathrm{~ms}$ epochs (0-400 ms and 400-800 ms) were chosen for the Stroop task, resulting in different but comparable time intervals for both tasks.

In order to compare the scalp topography of theta activity in the DMTS and the Stroop task and based on prior studies, we selected the time intervals, in which the demand on cognitive control and the measured theta activity were largest for the more difficult conditions (see Berger et al., 2016; Griesmayr et al., 2014; HansImayr et al., 2008 for a similar approach). In both tasks this was the case 
in late time intervals during the maintenance phase (1500-2000 ms for DMTS) and stimulus presentation (400-800 ms for Stroop), respectively. The extracted activity from all scalp electrodes was rescaled using the vector scaling method (McCarthy \& Wood, 1985; Picton et al., 2000; Wilding, 2006). This method was used to control for amplitude differences between conditions making the measured power values comparable across tasks, conditions, and electrodes. Therefore, difference scores of theta activity in both tasks ("manipulation - retention" at 1500-2000 ms for DMTS and "incongruent - congruent" at $400-800$ ms for Stroop) were calculated and averaged across participants. The 25 electrodes used for the vector scaling method were Fp1, Fp2, F7, F3, Fz, F4, F8, FC5, FC3, FCz, FC4, FC6, T7, C3, Cz, C4, T8, CP3, CPz, CP4, P7, P3, Pz, P4, and P8.

\subsection{Data Analysis}

Behavioral effects on reaction times (RTs) and accuracy were assessed by one-way repeatedmeasures analyses of variance (ANOVAs) including the within-subject factor Condition (retention vs. manipulation for the DMTS task and congruent vs. neutral vs. incongruent for the Stroop task). FM theta effects at electrode Fz were analyzed with repeated-measures ANOVAs. For the DMTS task the within-subject factors were Condition (retention vs. manipulation) and Time (0-500 ms vs. $500-1000$ ms vs. $1000-1500 \mathrm{~ms}$ vs. $1500-2000 \mathrm{~ms}$ vs. $2000-2500 \mathrm{~ms})$ whereas for the Stroop task the corresponding factors were Condition (congruent vs. incongruent) and Time (0-400 ms vs. $400-800$ ms), respectively. The neutral condition of the Stroop task was discarded from EEG analyses since on the basis of a large amount of other studies largest effects were expected for the comparison of the two classical Stroop conditions, congruent and incongruent respectively (e.g. Hanslmayr et al., 2008; see Macleod, 1991, for review). Additionally, this made the Stroop task analyses better comparable to the ones of the DMTS task. Between-task differences in the scalp distribution of theta ERS/ERD were assessed by a repeated-measures ANOVA with the within-subject factors task (DMTS vs. Stroop) and electrode ( 25 electrodes) conducted for the rescaled condition differences ("manipulation retention" for DMTS task and "incongruent - congruent" for Stroop task) in the 400 to $800 \mathrm{~ms}$ (Stroop task) and the 1500 to 2000 ms (DMTS task) time intervals, respectively. For all analyses, only 
correct trials were included and the significance level was set to $\alpha=.05$. Whenever necessary, the Greenhouse-Geisser correction was applied and the adjusted $p$-values are reported. When post-hoc comparisons were made, the Bonferroni correction method was applied in order to correct the $\alpha$ level for multiple comparisons.

\section{Results}

\subsection{Behavioral Results}

As expected and as illustrated by Table 1, in the DMTS task reaction times were faster and performance accuracy higher in the retention than in the manipulation condition. Furthermore, in the Stroop task participants responded fastest and most accurate in the congruent condition whereas performance was slower and less accurate in the other two conditions with slowest reaction times and lowest accuracy in the incongruent condition. These observations were confirmed by the statistical analyses. The one-way repeated-measures ANOVAs of the DMTS task revealed a main effect of Condition for reaction times $\left(F(1,26)=381.38, p<.001, \eta_{p}^{2}=.94\right)$ and for accuracy $(F(1,26)=$ $\left.253.81, p<.001, \mathrm{\eta}_{p}^{2}=.91\right)$. Analysis of the RTs in the Stroop task with a one-way repeated-measures ANOVA revealed a significant main effect of Condition $\left(F(2,66)=55.34, p<.001, \eta_{p}^{2}=.63\right)$ and posthoc t-tests with a Bonferroni-corrected $\alpha$-level of .017 showed that RTs in all three conditions differed significantly from each other with congruent $<$ incongruent $(t(33)=10.29, p<.001, d=1.76)$, neutral $<$ incongruent $(t(33)=6.00, p<.001, d=1.03)$, and congruent $<$ neutral $(t(33)=4.61, p<.001$, $d=0.79)$, reflecting increasing demands on interference resolution from congruent over neutral to incongruent trials. Differences in accuracy between Stroop conditions were revealed by a significant main effect of Condition $\left(F(2,66)=9.06, p=.001, \eta_{p}^{2}=.22\right)$. Further t-tests showed that with a Bonferroni-corrected $\alpha$-level of .017 the congruent $>$ incongruent $(t(33)=3.25, p=.003, d=0.56)$ and neutral $>$ incongruent $(t(33)=3.43, p=.002, d=0.59)$ differences were significant whereas the difference between the congruent and neutral condition was not $(t(33)=0.34, p=.733, d=0.06)$, indicating that an interference effect (incongruent < neutral) but not a facilitation effect (congruent > neutral) was present in the accuracy data (see Macleod, 1991, for review). In sum, consistent with 
our hypotheses, performance was slower and less accurate in conditions that require increased cognitive control for the active maintenance and manipulation of information in visual WM (DMTS task) or the inhibition of a prepotent response in favor of a less preferred one (Stroop task), reflecting high demands on proactive and reactive cognitive control, respectively.

\section{Table 1}

Behavioral results of the DMTS $(n=27)$ and Stroop task $(n=34)$.

\begin{tabular}{lllll} 
Task & Condition & Reaction Times [ms] & Accuracy [\%] & Difficulty \\
\hline \multirow{2}{*}{ DMTS } & retention & $684(24)$ & $97.94(.43)$ & low \\
& manipulation & $1059(32)$ & $73.97(1.45)$ & high \\
\hline \multirow{2}{*}{ Stroop } & congruent & $601(9)$ & $92.14(.84)$ & low \\
& neutral & $619(9)$ & $91.87(.83)$ & medium \\
& incongruent & $640(10)$ & $88.05(1.47)$ & high \\
\hline
\end{tabular}

Note. Standard errors of the mean are depicted in parentheses. Each task contained conditions varying in difficulty and thus in the need for cognitive control.

\subsection{EEG Results}

For the DMTS task the repeated-measures ANOVA with the within-subject factors Condition (retention vs. manipulation) and Time (0-500 ms vs. $500-1000 \mathrm{~ms}$ vs. $1000-1500 \mathrm{~ms}$ vs. $1500-2000 \mathrm{~ms}$ vs. 2000-2500 ms) conducted for FM theta ERS/ERD showed a significant Condition by Time interaction $\left(F(2.14,55.628)=4.19, p=.018, \mathrm{\eta}_{p}^{2}=.14\right)$, indicating that theta activity changed differently over time for both conditions. Both main effects were not significant ( $p$-values $>.056$ ). As apparent from Figure 2 and confirmed by further t-tests with a Bonferroni corrected $\alpha$-level of .01, theta ERS was marginally higher in the manipulation condition than in the retention condition during the maintenance phase in the $1500-2000$ ms time interval $(t(26)=1.84, p=.039, d=0.72)$. Other comparisons between conditions were not significant (all $p$-values $>.069$ ). Further contrasts for the factor Time, that were calculated separately for both conditions, revealed that in the retention condition FM theta activity decreased linearly over the time intervals of the encoding and 
maintenance phase $\left(F(1,26)=10.67, p=.003, \eta_{p}^{2}=.29\right)$ whereas in the manipulation condition the linear trend was not significant $\left(F(1,26)=.055, p=.817, \eta_{p}^{2}=.002\right)$. These findings suggest that in the retention condition cognitive control demands are high in the initial encoding phase and then decline during the maintenance phase whereas in the manipulation condition the demands on cognitive control are relatively stable across time.

A
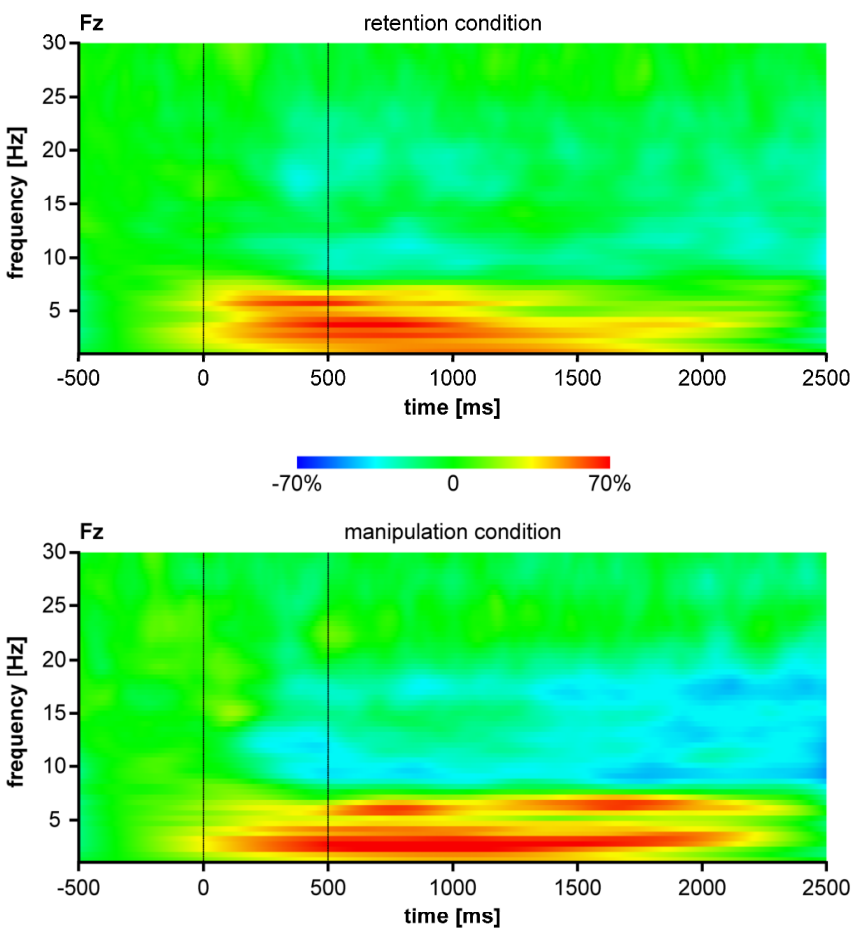

B

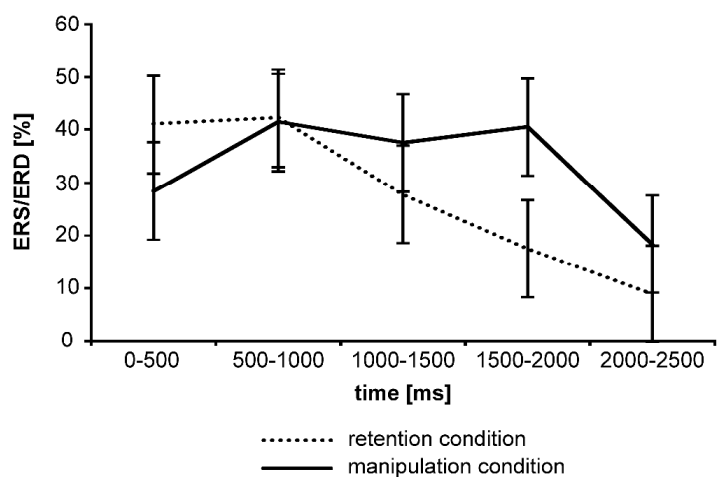

Stroop task
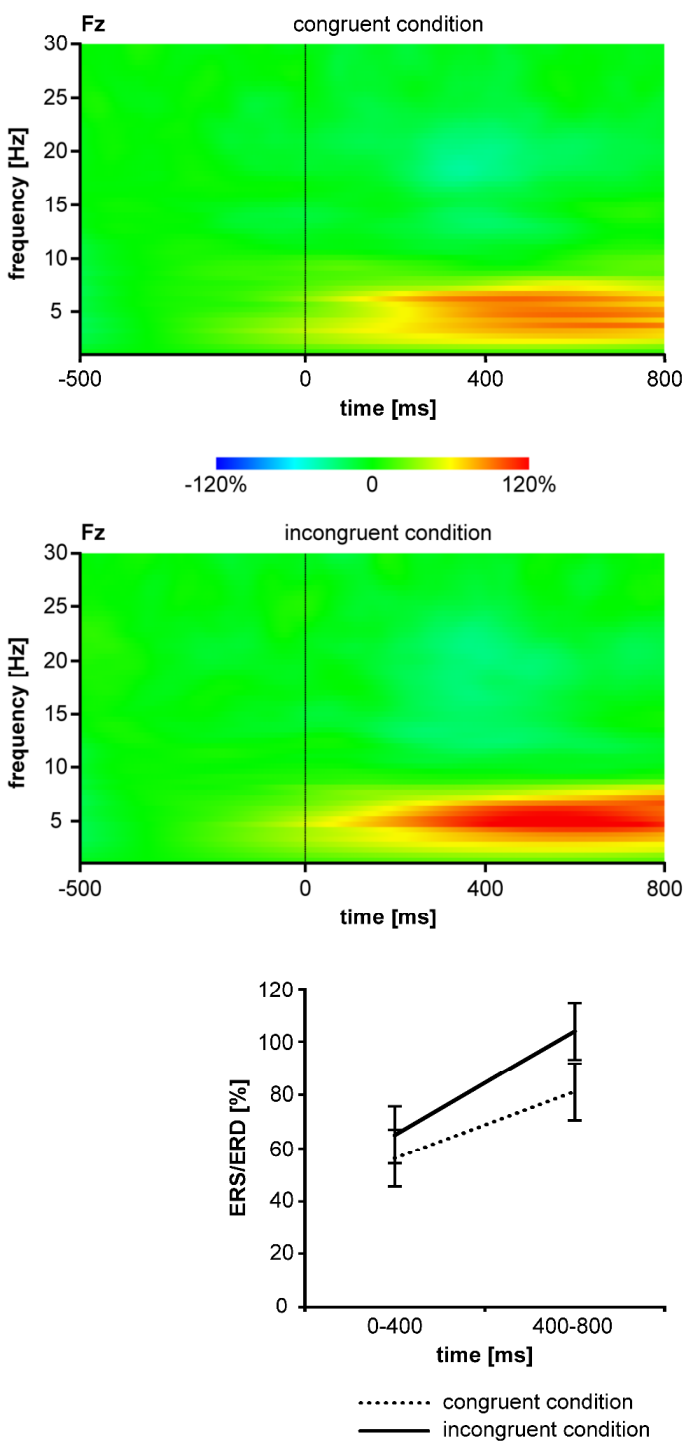

Figure. 2. (A) Time-frequency plots of ERS/ERD activity for the retention and manipulation condition of the DMTS task $(n=27)$ and the congruent and incongruent condition of the Stroop task $(n=34)$ at electrode Fz. Vertical bars indicate stimulus and delay onset, respectively. For both tasks a $300 \mathrm{~ms}$ baseline was used. (B) Mean FM theta activity of the consecutive time intervals for the conditions of the DMTS and Stroop task at electrode Fz. Error bars indicate 95\% confidence interval of the Condition by Time interaction for each task. 
Analysis of the FM theta ERS/ERD in the Stroop task with a repeated-measures ANOVA including the within-subject factors Condition (congruent vs. incongruent) and Time (0-400 ms vs. $400-800 \mathrm{~ms})$ revealed both a significant main effect of Condition $\left(F(1,33)=52.48, p<.001, \eta_{p}^{2}=.61\right)$ and of Time $\left(F(1,33)=9.11, p=.005, \eta_{p}^{2}=.22\right)$. Furthermore, there was a significant Condition by Time interaction $\left(F(1,33)=6.97, p=.013, \eta_{p}^{2}=.17\right)$. As depicted in Figure 2, t-tests with a Bonferronicorrected $\alpha$-level of .025 revealed that the congruent < incongruent effect was more pronounced in the late time interval $(t(33)=3.29, p=.001, d=1.15)$ than in the early time interval $(t(33)=2.17, p$ $=.019, d=0.76)$. This finding is in line with previous research showing that FM theta is especially pronounced in conditions with high need for cognitive control, such as the incongruent Stroop condition, and occurs in a late response-selection phase of the task, in which the prepotent wordreading response has to be inhibited.

The comparison of condition differences (manipulation - retention at 1500-2000 ms for the DMTS task and incongruent - congruent at $400-800$ ms for the Stroop task) in the amplitude normalized theta scalp topography between both tasks with a repeated-measures ANOVA containing the within-subject factors Task (DMTS vs. Stroop) and Electrode (25 electrodes) revealed a significant Task by Electrode interaction $\left(F(4.053,105.374)=2.93, p=.024, \eta_{p}^{2}=.10\right)$. Figure 3 shows the topographical distribution of theta activity differences for both tasks across the 25 selected electrodes. The linear Task by Electrode interaction contrast was significant $(F(1,26)=5.848, p=.024$, $\left.\eta_{p}^{2}=.18\right)$, indicating a focal FM theta activation at frontal recordings that linearly declined from anterior to posterior recordings in the DMTS task as compared to a broad activation in the Stroop task. All in all, these findings show that although the condition effects were pronounced at the same electrode $\mathrm{Fz}$, the recruitment of proactive control in the DMTS task is reflected by a rather focal theta activation over frontal scalp sites whereas reactive control processes in the Stroop task are accompanied by a topographically more widespread theta activation. 


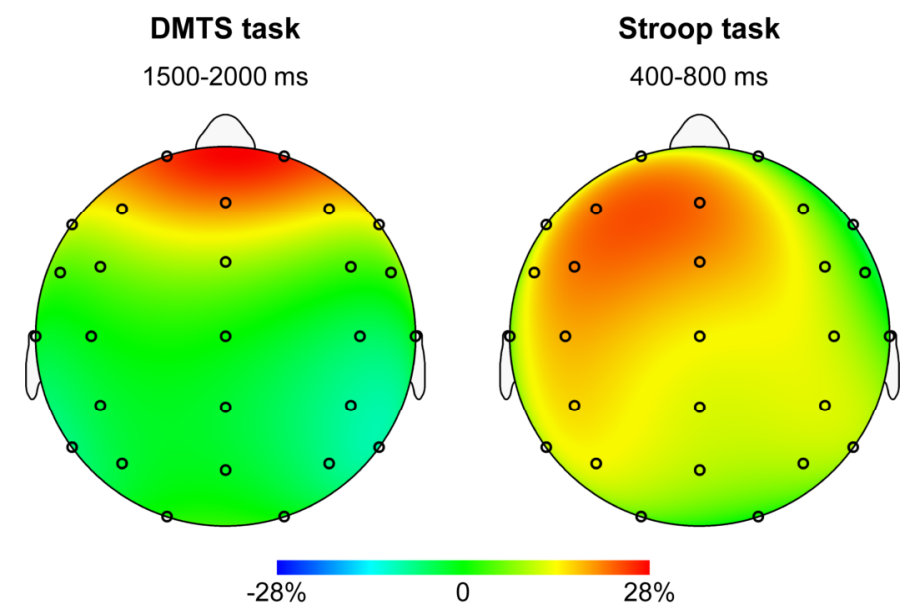

Figure. 3. Topographic maps of FM theta activity difference of the DMTS task (manipulation - retention) at $1500-2000 \mathrm{~ms}(\mathrm{n}=27)$ and Stroop task (incongruent congruent) at $400-800 \mathrm{~ms}(\mathrm{n}=27)$.

\section{Discussion}

The present study aimed at investigating the functional role of FM theta activity in two tasks requiring cognitive control. Both tasks contained conditions that differed in their difficulty and consequently in their cognitive control demands. Behavioral results revealed the validity of the difficulty manipulation of the conditions in each task. Participants showed slower and less accurate performance in conditions with high need for control, namely the manipulation condition of the DMTS task and the incongruent condition of the Stroop task (cf. Table 1). Moreover, we expected that the higher cognitive control demands in these conditions would be accompanied by higher FM theta compared to conditions with low control demands (cf. Cavanagh \& Frank, 2014). Analyses of the electrophysiological activity at electrode Fz supported this assumption by revealing different temporal profiles of FM theta activity across the encoding and maintenance phase for the two conditions of the DMTS task and higher FM theta activity in the incongruent than in the congruent condition of the Stroop task (cf. Figure 2). In the DMTS task, FM theta activity was high during the encoding phase in the retention condition and during the encoding and maintenance phase in the manipulation condition. This finding suggests different temporal trajectories of cognitive control in 
both conditions. While in the retention condition the internal representation of the stimulus can be build up immediately during encoding, in the manipulation condition a mirror transformation has to be performed first in order to construct an internal representation later in the maintenance period that can be matched with the upcoming probe. Consequently, FM theta might reflect cognitive control processes that serve both the construction (cf. Gruber et al., 2008; Khader et al., 2010; Osipova et al., 2006; Sederberg et al., 2007; see Nyhus \& Curran, 2010, for review) and maintenance (Griesmayr, Gruber, Klimesch, \& Sauseng, 2010; Roberts, Hsieh, \& Ranganath, 2014; see Sauseng et al., 2010) of internal representations.

In the DMTS task the greatest difference in FM theta activity between the retention and manipulation condition was present in a later time window of the maintenance phase. Similarly, in the Stroop task FM theta was higher for the incongruent compared to the congruent condition in the later time interval where response-selection takes place and the prepotent word-reading response needs to be inhibited. Therefore, these time intervals were selected in order to investigate the specificity of theta activity as a neural correlate of cognitive control. It was expected that scalp topographical differences of theta oscillations might become apparent by directly comparing these two tasks which differ in the amount of proactive and reactive control, respectively. As expected, the distribution of theta activity over scalp electrodes differed significantly between both tasks. Theta activity in the DMTS task was strongest at frontal electrodes with decreasing theta activity from anterior to posterior sites whereas theta activity was topographically more broadly distributed in the Stroop task, as indicated by a linear interaction contrast in the amplitude normalized data. This result can be interpreted in the context of the DMC framework (Braver, 2012) that states that proactive control is reflected in sustained IPFC activation (Braver \& Bongiolatti, 2002; Braver \& Cohen, 2001; Paxton et al., 2007; Veltman et al., 2003) whereas reactive control recruits IPFC and a wider network of brain areas (Grandjean et al., 2012; Milham et al., 2002; Zysset et al., 2001). The focal theta activation over prefrontal brain regions in the DMTS task probably reflects proactive control mode that supports the sustained maintenance of stimulus representations and facilitates the processing of 
upcoming events, such as the probe. In contrast, the broad theta distribution in the Stroop task might reflect the reactive control mode that enables a late correction process that happens after situations characterized by high response conflict and thereby supports interference resolution. Consequently, the broader distributed theta scalp topography in the Stroop task as compared to the focal theta activity in the DMTS task might originate from the activation and synchronization of broadly distributed brain regions. Although this result shows only indirect evidence for large scale theta synchronization, the present interpretation is partly supported by recent theta coherence studies that show the activation of different fronto-parietal theta networks during proactive compared to reactive control (Cooper et al., 2015, 2017). However, Cooper et al. $(2015,2017)$ investigated transient changes in proactive control in the trial-preparation phase of a task-switching paradigm. By this, one cannot infer from their findings that the same network is involved in sustained proactive control (Cooper et al., 2015) that was investigated in the maintenance phase of the DMTS task in the present study.

Taken into account that FM theta activity was modulated in amplitude and topography by cognitive control demands in both tasks, the question arises which mechanisms are reflected in theta oscillations that serve both proactive and reactive control. In a recent review paper, Hsieh and Ranganath (2014) proposed that FM theta oscillations might organize the sequential reactivation of individual items in WM that also supports long-term memory encoding and retrieval. Individual items are reflected by gamma activity that is superimposed onto one theta cycle by cross-frequency phase coupling and thus acquire a temporal order that can be sequentially reactivated (Jensen \& Lisman, 2005; Lisman \& Idiart, 1995; Lisman \& Jensen, 2013). Consequently, the more individual items have to be maintained in WM the more FM theta activity is present. In support of the view that frontal theta activity is especially important for the maintenance of temporal order information, Roberts et al. (2014) showed that frontal theta activity was more pronounced when temporal order as compared to spatial information was maintained in WM. The results of the DMTS task can be interpreted in line with the sequential reactivation view. In the manipulation condition, the four 
squares of the stimulus are probably encoded, mirrored at the vertical stimulus midline and maintained sequentially and thus may be represented in WM by theta-gamma phase coupling. Consistently, theta activity was higher in the manipulation condition, in which participants had to manipulate and maintain four square positions of the stimulus, as compared to the retention condition, in which only one square position had to be maintained. Additionally, the difference between conditions in theta activity was especially pronounced at frontal sides. Thus, it is conceivable that especially the focally measured FM theta activity reflects temporal order information about the encoded stimuli.

Another related account for FM theta activity, that is also discussed by Hsieh and Ranganath (2014), assumes that FM theta is involved in the systematic coordination of inhibition strength over strong and weak representations (Norman, Newman, Detre, \& Polyn, 2006; Norman, Newman, \& Perotte, 2005). It is assumed that inhibition levels vary with the theta cycle and thereby support the strengthening of weak target representations while suppressing strong competitors. In both tasks of the present study, inhibition plays a role for successful task performance. In the DMTS task, competing stimulus representations of previous trials and not yet mirrored stimulus representations of the present trial have to be inhibited during WM maintenance and the subsequent comparison with the probe. Thus, FM theta activity could proactively support the processing of the probe in a top-down manner by strengthening the task-relevant stimulus representation and inhibiting competing representations. However, inhibition strength probably is not as strong as in the Stroop task since there is no prepotent stimulus or response representation that needs to be inhibited. In the color Stroop task, the response representation of naming the color word erroneously gets activated first and needs to be inhibited reactively in order to overcome interference and to name the word color. Therefore, the broad theta scalp distribution in the Stroop task probably reflects the inhibition of strong prepotent representations while supporting the weak task goal representation. Taken together, both accounts can explain the findings in the present study. Although the DMTS task involves aspects that can be explained by the account of inhibition strength coordination, the results 
of the DMTS task are best explained by the sequential reactivation account due to the sequential nature of the task. In contrast, the results of the Stroop task can be best interpreted in line with the account of theta activity as a coordination mechanism of inhibition strength due to larger interference in the Stroop compared to the DMTS task.

Although the findings of the present study can be interpreted in line with the dual mechanisms framework (Braver, 2012) and provide evidence for the view that theta can support different control functions depending on task requirements, some limitations of the present study have to be considered. Given that our analyses were exploratory in nature, these limitations might be addressed by future studies in order to substantiate the research on differential theta control networks. The tasks of the present study were not intentionally designed to specifically compare proactive and reactive processes reflected in theta amplitude and its topographical distribution. Both tasks were part of a larger study that additionally contained an episodic retrieval task and aimed at investigating the functional role of FM theta activity within these tasks. One might argue that theta differences between tasks are due to differences in task material and procedure. However, since the topographical comparison between the two tasks is based on differences between task conditions, the influence of different task materials between the tasks should be excluded or at least substantially attenuated. A study in which both forms of cognitive control are operationalized with the same verbal or visuo-spatial task material would directly address this latter issue and should shed further light on this topic. As in other studies the DMTS and Stroop task are not process-pure measures of proactive and reactive control processes, respectively, but recruit the one or the other processes to a greater extent. Moreover, in the DMTS task, task difficulty was defined by both task instruction and WM load whereas in the Stroop task only in/congruency of word and ink color determined task difficulty. Therefore, when discussing the functional role of theta activity, it has to be considered that the present results reflect a relative difference in cognitive control demands due to general task difficulty. However, both tasks are tasks that are typically used in investigations of the dual mechanisms of control framework (cf. Braver, 2012). 
All in all, to the best of our knowledge the present study is the first that shows scalp topographical differences of theta activity between tasks differing in their cognitive control demands, demonstrating the importance of taking scalp topographical differences into account in the functional interpretation of FM theta activity. In line with Hsieh \& Ranganath (2014), the present study supports the view that FM theta activity may reflect either the communication of temporal order information by sequential reactivation of items in WM or the coordination of inhibition strength, depending on the task requirements. FM theta oscillations might inherit one or the other of these functional roles depending on the either proactive or reactive cognitive control demands of the task.

\section{Acknowledgements}

The authors would like to thank Paul Sauseng for providing the stimuli of the DMTS task and Johannes J. Bürkle, Hong-Hanh Pham and Juliana Koch for their help during data collection. This research was supported by the German Research Foundation under grant GRK 1457 and was conducted within the International Research Training Group "Adaptive Minds" hosted by Saarland University, Department of Psychology, Saarbrücken (Germany) and the Chinese Academy of Sciences, Institute of Psychology, Beijing (China) as part of K.C.J.E.'s dissertation project.

\section{Conflict of interest}

The authors declare no conflicts of interest.

\section{References}

Asada, H., Fukuda, Y., Tsunoda, S., Yamaguchi, M., \& Tonoike, M. (1999). Frontal midline theta rhythms reflect alternative activation of prefrontal cortex and anterior cingulate cortex in humans. Neuroscience Letters, 274(1), 29-32. doi: 10.1016/S0304-3940(99)00679-5

Berger, B., Minarik, T., Griesmayr, B., Stelzig-Schoeler, R., Aichhorn, W., \& Sauseng, P. (2016). Brain oscillatory correlates of altered executive functioning in positive and negative symptomatic schizophrenia patients and healthy controls. Frontiers in Psychology, 7, 1-14. doi: 10.3389/fpsyg.2016.00705

Braver, T. S. (2012). The variable nature of cognitive control: a dual mechanisms framework. Trends 
in Cognitive Sciences, 16(2), 106-113. doi: 10.1016/j.tics.2011.12.010

Braver, T. S., \& Bongiolatti, S. R. (2002). The role of frontopolar cortex in subgoal processing during working memory. Neurolmage, 15(3), 523-536. doi: doi.org/10.1006/nimg.2001.1019

Braver, T. S., \& Cohen, J. D. (2001). Working memory, cognitive control, and the prefrontal cortex: computational and empirical studies. Cognitive Processing, 2(1), 2555. Retrieved from http://ccpweb.wustl.edu/pdfs/CP.pdf

Cavanagh, J. F., \& Frank, M. J. (2014). Frontal theta as a mechanism for cognitive control. Trends in Cognitive Sciences, 18(8), 414-421. doi: 10.1016/j.tics.2014.04.012

Cavanagh, J. F., Frank, M. J., Klein, T. J., \& Allen, J. J. B. (2010). Frontal theta links prediction errors to behavioral adaptation in reinforcement learning. Neurolmage, 49(4), 3198-3209. doi: 10.1016/j.neuroimage.2009.11.080

Cavanagh, J. F., Zambrano-Vazquez, L., \& Allen, J. J. B. (2012). Theta lingua franca: a common midfrontal substrate for action monitoring processes. Psychophysiology, 49(2), 220-238. doi: 10.1111/j.1469-8986.2011.01293.x

Cohen, M. X. (2011). Error-related medial frontal theta activity predicts cingulate-related structural connectivity. Neurolmage, 55(3), 1373-1383. doi: 10.1016/j.neuroimage.2010.12.072

Cohen, M. X., van Gaal, S., Ridderinkhof, K. R., \& Lamme, V. A. F. (2009). Unconscious errors enhance prefrontal-occipital oscillatory synchrony. Frontiers in Human Neuroscience, 3, 54. doi: 10.3389/neuro.09.054.2009

Cooper, P. S., Wong, A. S. W., Fulham, W. R., Thienel, R., Mansfield, E., Michie, P. T., \& Karayanidis, F. (2015). Theta frontoparietal connectivity associated with proactive and reactive cognitive control processes. Neurolmage, 108, 354-363. doi: 10.1016/j.neuroimage.2014.12.028

Cooper, P. S., Wong, A. S. W., McKewen, M., Michie, P. T., \& Karayanidis, F. (2017). Frontoparietal theta oscillations during proactive control are associated with goal-updating and reduced behavioral variability. Biological Psychology, 129, 253-264. doi: 10.1016/j.biopsycho.2017.09.008

David, O., Kilner, J. M., \& Friston, K. J. (2006). Mechanisms of evoked and induced responses in MEG/EEG. Neurolmage, 31(4), 1580-1591. doi: 10.1016/j.neuroimage.2006.02.034

Ferreira, C. S., Marful, A., Staudigl, T., Bajo, T., \& Hanslmayr, S. (2014). Medial prefrontal theta oscillations track the time course of interference during selective memory retrieval. Journal of Cognitive Neuroscience, 26(4), 777-791. doi: 10.1162/jocn_a_00523 
Gevins, A., Smith, M. E., McEvoy, L., \& Yu, D. (1997). High-resolution EEG mapping of cortical activation related to working memory: Effects of task difficulty, type of processing, and practice. Cerebral Cortex, 7(4), 374-385. doi: 10.1093/cercor/7.4.374

Grandjean, J., Ostilio, K. D., Phillips, C., Balteau, E., Degueldre, C., Maquet, P., ... Collette, F. (2012). Modulation of brain sctivity during a Stroop inhibitory task by the kind of cognitive control required. PLOS ONE, 7(7), e41513. doi: 10.1371/journal.pone.0041513

Griesmayr, B., Berger, B., Stelzig-Schoeler, R., Aichhorn, W., Bergmann, J., \& Sauseng, P. (2014). EEG theta phase coupling during executive control of visual working memory investigated in individuals with schizophrenia and in healthy controls. Cognitive, Affective \& Behavioral Neuroscience, 14(4), 1340-1355. doi: 10.3758/s13415-014-0272-0

Griesmayr, B., Gruber, W. R., Klimesch, W., \& Sauseng, P. (2010). Human frontal midline theta and its synchronization to gamma during a verbal delayed match to sample task. Neurobiology of Learning and Memory, 93(2), 208-215. doi: 10.1016/j.nIm.2009.09.013

Gruber, T., Tsivilis, D., Giabbiconi, C.-M., \& Müller, M. M. (2008). Induced electroencephalogram oscillations during source memory: familiarity is reflected in the gamma band, recollection in the theta band. Journal of Cognitive Neuroscience, 20(6), 1043-1053. doi: 10.1162/jocn.2008.20068

Hanslmayr, S., Pastötter, B., Bäuml, K.-H., Gruber, S., Wimber, M., \& Klimesch, W. (2008). The electrophysiological dynamics of interference during the Stroop task. Journal of Cognitive Neuroscience, 20(2), 215-25. doi: 10.1162/jocn.2008.20020

Helfrich, R. F., \& Knight, R. T. (2016). Oscillatory dynamics of prefrontal cognitive control. Trends in Cognitive Sciences, 20(12), 916-930. doi: 10.1016/j.tics.2016.09.007

Hsieh, L. T., \& Ranganath, C. (2014). Frontal midline theta oscillations during working memory maintenance and episodic encoding and retrieval. Neurolmage, 85, 721-729. doi: 10.1016/j.neuroimage.2013.08.003

Jensen, O., \& Lisman, J. E. (2005). Hippocampal sequence-encoding driven by a cortical multi-item working memory buffer. Trends in Neurosciences, 28(2), 67-72. doi: 10.1016/j.tins.2004.12.001

Jensen, O., \& Tesche, C. D. (2002). Frontal theta activity in humans increases with memory load in a working memory task. European Journal of Neuroscience, 15(8), 1395-1399. doi: 10.1046/j.1460-9568.2002.01975.x

Kalcher, J., \& Pfurtscheller, G. (1995). Discrimination between phase-locked and non-phase-locked event-related EEG activity. Electroencephalography and Clinical Neurophysiology, 94(5), 381- 
384. doi: 10.1016/0013-4694(95)00040-6

Khader, P. H., Jost, K., Ranganath, C., \& Rösler, F. (2010). Theta and alpha oscillations during workingmemory maintenance predict successful long-term memory encoding. Neuroscience Letters, 468(3), 339-343. doi: 10.1016/j.neulet.2009.11.028

Klimesch, W., HansImayr, S., Sauseng, P., Gruber, W., Brozinsky, C. J., Kroll, N. E. a, ... Doppelmayr, M. (2006). Oscillatory EEG correlates of episodic trace decay. Cerebral Cortex, 16(2), 280-290. doi: 10.1093/cercor/bhi107

Lisman, J. E., \& Idiart, M. A. P. (1995). Storage of 7 plus/minus short-term memories in oscillatory subcycles. Science, 267(5203), 1512. doi: 10.1126/science.7878473

Lisman, J. E., \& Jensen, O. (2013). The theta-gamma neural code. Neuron, 77(6), 1002-1016. doi: 10.1016/j.neuron.2013.03.007

Macleod, C. M. (1991). Half a century of research on the Stroop effect: an integrative review. Psychological Bulletin, 109(2), 163-203. doi: 10.1037/0033-2909.109.2.163

McCarthy, G., \& Wood, C. C. (1985). Scalp distributions of event-related potentials: an ambiguity associated with analysis of variance models. Electroencephalography and Clinical Neurophysiology/Evoked Potentials Section, 62(3), 203-208. doi: 10.1016/0168-5597(85)900152

Milham, M. P., Erickson, K. I., Banich, M. T., Kramer, A. F., Webb, A., Wszalek, T., \& Cohen, N. J. (2002). Attentional control in the aging brain: insights from an fMRI study of the Stroop task. Brain and Cognition, 49(3), 277-296. doi: 10.1006/brcg.2001.1501

Narayanan, N. S., Cavanagh, J. F., Frank, M. J., \& Laubach, M. (2013). Common medial frontal mechanisms of adaptive control in humans and rodents. Nature Neuroscience, 16(12), 18881895. doi: $10.1038 / \mathrm{nn} .3549$

Niendam, T. A., Laird, A. R., Ray, K. L., Dean, Y. M., Glahn, D. C., \& Carter, C. S. (2012). Meta-analytic evidence for a superordinate cognitive control network subserving diverse executive functions. Cognitive, Affective, \& Behavioral Neuroscience, 12(2), 241-268. doi: 10.3758/s13415-011-00835

Nigbur, R., Ivanova, G., \& Stürmer, B. (2011). Theta power as a marker for cognitive interference. Clinical Neurophysiology, 122(11), 2185-2194. doi: 10.1016/j.clinph.2011.03.030

Norman, K. A., Newman, E., Detre, G., \& Polyn, S. (2006). How inhibitory oscillations can train neural networks and punish competitors. Neural Computation, 18(7), 1577-1610. doi: 10.1162/neco.2006.18.7.1577 
Norman, K. A., Newman, E. L., \& Perotte, A. J. (2005). Methods for reducing interference in the complementary learning systems model: oscillating inhibition and autonomous memory rehearsal. Neural Networks, 18(9), 1212-1228. doi: 10.1016/j.neunet.2005.08.010

Nyhus, E., \& Curran, T. (2010). Functional role of gamma and theta oscillations in episodic memory. Neuroscience and Biobehavioral Reviews, 34(7), 1023-1035. doi: 10.1016/j.neubiorev.2009.12.014

Onton, J., Delorme, A., \& Makeig, S. (2005). Frontal midline EEG dynamics during working memory. Neurolmage, 27(2), 341-356. doi: 10.1016/j.neuroimage.2005.04.014

Osipova, D., Takashima, A., Oostenveld, R., Fernández, G., Maris, E., \& Jensen, O. (2006). Theta and gamma oscillations predict encoding and retrieval of declarative memory. Journal of Neuroscience, 26(28), 7523-7531. doi: 10.1523/JNEUROSCI.1948-06.2006

Paxton, J. L., Barch, D. M., \& Racine, C. A. (2007). Cognitive control, goal maintenance, and prefrontal function in healthy aging. Cerebral Cortex, 18(5), 1010-1028. doi: 10.1093/cercor/bhm135

Picton, T. W., Bentin, S., Berg, P., Donchin, E., Hillyard, S. A., Johnson, R., ... Ruchkin, D. S. (2000). Guidelines for using human event-related potentials to study cognition: recording standards and publication criteria. Psychophysiology, 37(2), 127-152. doi: 10.1111/1469-8986.3720127

Rebai, M., Bernard, C., \& Lannou, J. (1997). The Stroop's test evokes a negative brain potential, the N400. International Journal of Neuroscience, 91(1-2), 85-94. doi: 10.3109/00207459708986367

Roberts, B. M., Hsieh, L.-T., \& Ranganath, C. (2014). Oscillatory activity during maintenance of spatial and temporal information in working memory. Neuropsychologia, 51(2), 349-357. doi: 10.1016/j.neuropsychologia.2012.10.009

Sauseng, P., Griesmayr, B., Freunberger, R., \& Klimesch, W. (2010). Control mechanisms in working memory: a possible function of EEG theta oscillations. Neuroscience and Biobehavioral Reviews, 34(7), 1015-1022. doi: 10.1016/j.neubiorev.2009.12.006

Sederberg, P. B., Kahana, M. J., Howard, M. W., Donner, E. J., \& Madsen, J. R. (2003). Theta and gamma oscillations during encoding predict subsequent recall. Journal of Neuroscience, 23(34), 10809-10814. Retrieved from http://www.ncbi.nlm.nih.gov/pubmed/14645473

Sederberg, P. B., Schulze-Bonhage, A., Madsen, J. R., Bromfield, E. B., McCarthy, D. C., Brandt, A., ... Kahana, M. J. (2007). Hippocampal and neocortical gamma oscillations predict memory formation in humans. Cerebral Cortex, 17(5), 1190-1196. doi: 10.1093/cercor/bhl030

Spitzer, B., Hanslmayr, S., Opitz, B., Mecklinger, A., \& Bäuml, K.-H. (2009). Oscillatory correlates of retrieval-induced forgetting in recognition memory. Journal of Cognitive Neuroscience, 21(5), 
976-990. doi: 10.1162/jocn.2009.21072

Veltman, D. J., Rombouts, S. A. R. B., \& Dolan, R. J. (2003). Maintenance versus manipulation in verbal working memory revisited: an fMRI study. Neurolmage, 18(2), 247-256. doi: $10.1016 / \mathrm{S} 1053-8119(02) 00049-6$

Waldhauser, G. T., Bäuml, K.-H. T., \& Hanslmayr, S. (2014). Brain Oscillations Mediate Successful Suppression of Unwanted Memories. Cerebral Cortex, 25(11), 4180-4190. doi: 10.1093/cercor/bhu138

Wilding, E. L. (2006). The practice of rescaling scalp-recorded event-related potentials. Biological Psychology, 72(3), 325-332. doi: 10.1016/j.biopsycho.2005.12.002

Wilson, G. F., Swain, C. R., \& Ullsperger, P. (1999). EEG power changes during a multiple level memory retention task. International Journal of Psychophysiology, 32(2), 107-118. doi: 10.1016/S0167-8760(99)00005-7

Zysset, S., Mu, K., Lohmann, G., \& Cramon, D. Y. Von. (2001). Color-word matching Stroop task: separating interference and response conflict. Neurolmage, 36(1), 29-36. doi: 10.1006/nimg.2000.0665 